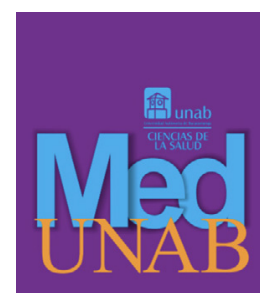

REVISTA DE LA FACULTAD

DE CIENCIAS DE LA SALUD

\title{
Análisis en el uso del condón femenino en estudiantes de una universidad privada de Cali-Colombia
}

Analysis of the use of the female condom in students of a private university in Cali, Colombia

Análise do uso do preservativo feminino em estudantes de uma universidade privada em Cali-Colômbia

Sonia Quebradas-Jiménez, Enf., MSP. ${ }^{1}$ D , Estela Rodríguez-Torres, Enf., MSO. ${ }^{2}$ D

1. Enfermera, Maestría en Salud Pública, Docente Universidad Santiago de Cali, Cali, Colombia.

2. Enfermera, Maestría en Salud Ocupacional, Docente Universidad Santiago de Cali, Cali, Colombia.

Correspondencia. Estela Rodríguez Torres. Universidad Santiago de Cali. Dirección. Calle 3C 63A-44 Sector C. Email.estelaroto@hotmail.com

\section{INFORMACIÓN DEL ARTÍCULO:}

Artículo recibido: 19 de junio de 2020

Artículo aceptado: 17 de junio de 2021

DOI: $\underline{\text { https://doi.org/10.29375/01237047.3930 }}$

Cómo citar. Quebradas-Jiménez S, Rodríguez-Torres E. Análisis en el uso del condón femenino en estudiantes de una universidad privada de Cali-Colombia. MedUNAB. 2021; 24(2):183-192 doi: https://doi. org $/ 10.29375 / 01237047.3930$

\section{RESUMEN}

Introducción. El condón femenino es un método de barrera eficaz para prevenir las enfermedades de transmisión sexual y los embarazos, permite la mejora de la salud sexual y reproductiva de las mujeres y sus parejas. Presenta barreras al utilizarse en aspectos socioculturales, económicos, religiosos y psicológicos, los cuales pueden ser superados a través de la promoción y educación. El objetivo del presente artículo es analizar el uso del condón femenino en estudiantes universitarios de un programa de salud. Metodología. Estudio cuasiexperimental, con muestreo por conveniencia realizado en 82 estudiantes, organizados en un grupo de 74 mujeres y otro de 8 hombres, quienes utilizaron el condón femenino en sus relaciones sexuales durante un período de tres meses. Reconocieron las ventajas, desventajas, sensaciones y reacciones de la pareja. Resultados. Las ventajas establecidas por todos los hombres y el $70 \%$ de las mujeres es colocar el condón femenino antes de la relación sexual, del mismo modo, el $39.2 \%$ de las mujeres y el $37.2 \%$ de los hombres indican como ventaja poder usarlo hasta el final de la relación. Las desventajas identificadas comprenden que el $54.1 \%$ de las parejas de las mujeres no les gusta utilizarlo, al $40.5 \%$ de las 
mujeres les parece difícil su colocación, y al 33.8\% le disminuye el placer sexual. En los hombres se identifican como desventajas la disminución del placer en la relación sexual, al $87.5 \%$ de sus parejas no le gusta utilizarlo, y el $62.5 \%$ de los hombres expresa dificultad para su colocación. Discusión. El uso del condón femenino está limitado por las condiciones de acceso y disponibilidad. El procedimiento de inserción del dispositivo en las mujeres requiere entrenamiento previo e información específica para su uso. Conclusión. La utilización del condón femenino es una alternativa viable para tener sexo seguro, los hombres presentan mayor rechazo al verse afectado el placer sexual.

Palabras clave:

Dispositivos Anticonceptivos; Sexo Seguro; Anticoncepción de Barrera; Conductas Relacionadas con la Salud; Salud Sexual.

\section{ABSTRACT}

Introduction. The female condom is an effective barrier method to prevent sexually transmitted diseases and pregnancy; it allows the improvement of the sexual and reproductive health of women and their partners. Using it presents barriers in sociocultural, economic, religious and psychological aspects, which can be overcome through promotion and education. The objective of this article is to analyze the use of the female condom in university students of a health program. Methodology. Quasi-experimental study, with convenience sampling carried out in 82 students, organized in a group of 74 women, and another of 8 men, who used the female condom in their sexual intercourse during a period of three months. They recognized the advantages, disadvantages, feelings and reactions of the partner. Results. The advantages established by all men and $70 \%$ of women is to place the female condom before sexual intercourse; in the same way, $39.2 \%$ of women and $37.2 \%$ of men indicate as an advantage the possibility to use it until the end of the intercourse. The identified disadvantages include that $54.1 \%$ of women's partners do not like to use it, $40.5 \%$ of women find it difficult to insert, and for $33.8 \%$ of them, it decreases sexual pleasure. In men, the disadvantages identified were the decrease in pleasure in sexual intercourse, $87.5 \%$ of their partners do not like to use it, and $62.5 \%$ of men express difficulty in inserting it. Discussion. The use of the female condom is limited by the conditions of access and availability. The procedure for inserting the device in women requires prior training and specific information for its use. Conclusion. The use of the female condom is a viable alternative to have safe sex. Men show greater rejection when sexual pleasure is affected.

Keywords:

Contraceptive Devices; Safe Sex; Barrier Contraception; Health Related Behaviors; Sexual Health.

\section{RESUMO}

Introdução. O preservativo feminino é um método de barreira eficaz na prevenção de doenças sexualmente transmissíveis e gravidez, melhorando a saúde sexual e reprodutiva da mulher e dos seus parceiros. Apresenta limitações no seu uso com relação a aspectos socioculturais, econômicos, religiosos e psicológicos, que podem ser superadas por meio de promoção e educação. O objetivo deste artigo é analisar o uso do preservativo feminino em estudantes de universidade de um programa de saúde. Metodologia. Estudo quase experimental, com amostragem por conveniência realizada em 82 estudantes, organizados em um grupo de 74 mulheres e outro de 8 homens, que usaram o preservativo feminino nas suas relações sexuais durante um período de três meses. Eles reconheceram as vantagens, desvantagens, sensações e reações do casal. Resultados. As vantagens estabelecidas por todos os homens e $70 \%$ das mulheres é colocar o preservativo feminino antes da relação sexual, da mesma forma, 39.2\% das mulheres e 37.2\% dos homens apontam como vantagem poder usá-lo até o fim da relação sexual. As desvantagens identificadas incluem que 54.1\% dos parceiros das mulheres não gostam de usá-lo, 40.5\% das mulheres têm dificuldade em colocá-lo e $33.8 \%$ acham que diminui o prazer sexual. Nos homens, identificam-se como desvantagens diminuição do prazer na relação sexual, $87.5 \%$ das parceiras não gostam de usá-lo e $62.5 \%$ dos homens expressam dificuldade em colocá-lo. Discussão. O uso do preservativo feminino é limitado pelas condições de acesso e disponibilidade. O procedimento de inserção do dispositivo em mulheres requer treinamento prévio e informações específicas para seu uso. Conclusão. O uso do preservativo feminino é uma alternativa viável para a prática de sexo seguro, os homens apresentam maior rejeição quando o prazer sexual é afetado.

Palavras-chave:

Dispositivos Contraceptivos; Sexo Seguro; Contracepção de Barreira; Comportamentos relacionados à Saúde; Saúde Sexual. 


\section{Introducción}

El condón femenino $(\mathrm{CF})$ es un método de barrera eficaz, previene el embarazo de las mujeres y las infecciones de transmisión sexual (ITS), les brinda autonomía sin tener que convencer a sus parejas de su uso, permitiéndoles cuidar su salud (1).

A diferencia del condón masculino que se ajusta al pene, el CF puede ser colocado en la vagina mucho antes de la relación sexual, evitando la interrupción de la que constantemente se quejan las parejas frente al condón masculino, que requiere la erección para ponerlo.

Es comercializado con el nombre de Female Condón, introducido en más de 90 países, avalado y promocionado por la Organización Mundial de la Salud (OMS), agregado a la lista de adquisiciones del Fondo de Población de las Naciones Unidas (UNFPA) (2).

Ha sido empleado para la prevención del Síndrome de Inmunodeficiencia Adquirida (SIDA) $(3,4)$. En todo el mundo, según ONUSIDA, la distribución de preservativos ha aumentado de forma sustancial en los últimos años, pero sigue existiendo una gran brecha en los países de bajos y medianos ingresos, está un $40 \%$ por debajo de las necesidades (5).

En el 2013 se realizó una investigación para comparar la aceptabilidad de dos tipos de CF: el Condón Femenino Phoenurse (PFC) y Condón Femenino Femidom (FC2) entre las mujeres chinas. Los resultados indicaron antes de la participación en el estudio que, de 290 mujeres, $247(85.2 \%)$ cree que el CF podría proteger contra las ITS y el VIH, $152(52.5 \%)$ cree que la tasa de fracaso como anticonceptivo del CF fue mayor, y 107 (36.9\%) cree que podría ser reutilizado. Después del estudio, $135(46.6 \%)$ y 39 mujeres (13.4\%) habrían estado dispuestos a usar el FC2 y el PFC, respectivamente (6).

Los proveedores de cuidado en salud en Sudáfrica evidenciaron con respecto al tamaño y la forma del condón femenino de segunda generación la dificultad para su inserción en los genitales femeninos, y a su vez, lo doloroso e incómodo que resulta durante el coito (7). La necesidad de un trabajo multidisciplinario en la educación y entrenamiento en cuidados primarios en su utilización debe orientar para anticiparse o atender tempranamente aquellas situaciones que desde las primeras experiencias pudieran convertirse en factores para el rechazo, tal como lo encontrado en la provincia de Gauteng Tshwane de África (8).
En la población universitaria de España, las mujeres enfatizan, en mayor medida que los varones, la satisfacción y ventajas obtenidas con la utilización del método, destacan la alta resistencia a la rotura; entre las debilidades apuntan los problemas ligados a su colocación y estética (1).

La aceptación y el conocimiento del uso del CF varía de acuerdo con el grupo poblacional, en la población migrante de Costa Rica, un grupo de mujeres estarían dispuestas a probar el CF a corto plazo (10.9\%) (9). Entre 318 estudiantes de enfermería en Ambato, Ecuador, el 94.8\% manifestaron haber oído hablar del $\mathrm{CF}$, pero nunca lo han usado (10).

Otros aspectos a tener en cuenta en el empleo del $\mathrm{CF}$ es la identificación de las barreras para el acceso y uso del condón, en una investigación llevada a cabo en la Habana, se expresa la necesidad de superar, principalmente, las barreras socioculturales y estructurales que frenan los esfuerzos de prevención en el mercadeo social del condón y en las acciones de prevención de las ITS/VIH/SIDA (11).

En Colombia, a pesar de los avances en el número de mujeres que planifican, aún existe una necesidad insatisfecha de anticoncepción (12). El empleo de anticonceptivos modernos se ha incrementado. En 1990, el $54.6 \%$ de mujeres de edades entre los 15 y 49 años empleaban anticonceptivos, para el 2015 el porcentaje aumentó a $75.9 \%$, lo que ha tenido un impacto en la reducción de la natalidad $(12,13)$. Sin embargo, la utilización de los anticonceptivos modernos no ha mejorado los indicadores con respecto a la protección para la presencia de la sífilis gestacional y congénita (14).

Para su implementación en los servicios de salud existe la legislación respectiva (15), pero presenta barreras institucionales y de no disponibilidad acorde con el Plan de Salud Pública de Intervenciones Colectivas (PIC), el cual incluye el uso de los condones femeninos y masculinos en el listado de intervenciones, procedimientos, actividades e insumos a ejecutar en el PIC (16).

Los mayores apoyos para mejorar la utilización del CF y el masculino en Colombia se han dado por el Ministerio de Salud y Protección Social, y por el Fondo de Población de las Naciones Unidas - UNFPA, promocionando su utilización como una herramienta que mejora la salud sexual y reproductiva de las mujeres y sus parejas (17). El presentar disponibilidad del CF en el territorio permitió la realización del presente estudio, 
con el objetivo de analizar el uso del CF en estudiantes universitarios de un programa de salud, sus ventajas, desventajas, sensaciones y reacciones de sus parejas.

\section{Metolología}

Estudio cuasiexperimental, con muestreo por conveniencia realizado en 82 estudiantes universitarios de una universidad privada de Cali-Colombia. La muestra fue establecida a partir de la disponibilidad de los condones femeninos (3,000 CF), los cuales fueron donados por la Secretaría de Salud Pública Municipal, y el cumplimiento de los criterios de inclusión: pertenecer a un programa de pregrado de salud, no conocer sobre el CF, no haber tenido experiencia previa con el uso del $\mathrm{CF}$, contar con pareja en el momento del estudio, recibir entrenamiento previo en el uso del CF. El criterio de exclusión fue no completar el tiempo de utilización de tres meses del CF en sus relaciones sexuales.

Previo a la intervención, los investigadores explicaron los objetivos del estudio e hicieron énfasis en la autonomía para participar en él. Los estudiantes que aceptaron voluntariamente el estudio de intervención se encontraban entre IV y X semestre, debían cumplir con una capacitación previa acerca del CF sin ser aplicada evaluación previa.

La capacitación fue realizada por los investigadores, quienes explicaron las características del FC2 de nitrilo recomendado por la OMS, fueron proporcionadas explicaciones frente al uso del FC2 y los problemas más comunes que pueden presentarse (18), analizando las posibles soluciones con respecto a la introducción en el canal vaginal, manejo del anillo interno y la inserción completa alrededor del cuello uterino y su colocación en el introito vaginal. Se realizó demostración a través de simuladores anatómicos acerca de la inserción y extracción del FC2, al igual que fue empleada la exposición con material audiovisual. Se dio a conocer el dispositivo del FC2 con la descripción del fabricante, del mismo modo que las instrucciones para llevar a casa.

Los investigadores entregaron a cada participante dotación para el primer mes, quienes debían manifestar su aceptación de continuar para las entregas respectivas en el segundo y tercer mes de utilización, y posteriormente analizar el efecto del empleo del FC2, reconociendo las ventajas, desventajas, sensaciones en la relación sexual, sensaciones luego de la relación y reacciones de la pareja.
Se utilizó el instrumento elaborado por el Ministerio de Salud y Protección Social y el Fondo de Población de las Naciones Unidas (19), y otros estudios $(20,21)$, los cuales contemplan una encuesta autoadministrada, semi estructurada con un total de 21 preguntas organizadas en tres componentes: preguntas de información personal, régimen de afiliación al Sistema de Seguridad Social e información acerca del uso.

Los estudiantes fueron informados sobre el anonimato y confidencialidad de los datos y acerca de la realización del consentimiento informado, sin representar riesgo académico en la permanencia del estudiante en la universidad; el estudio tuvo aprobación del Comité de Ética y Bioética de la Facultad de Salud y autorización de las directivas para su realización, cumpliendo con la normatividad internacional de la Declaración de Helsinki (22) y la norma nacional para la realización de trabajos de investigación en salud de acuerdo con la Resolución 8430 de 1993 del Ministerio de Salud de Colombia (23).

Para analizar la información se utilizó estadística descriptiva, se empleó Epi Info 7, calculando frecuencias para todas las variables, incluyendo los intervalos de confianza de 95\% (IC 95\%), se utilizó la prueba Chi cuadrado (X2) para establecer la significación del estimador en relación con otras variables, se consideró significativo un valor $\mathrm{p}<0.05$.

\section{Resultados}

La edad de los participantes oscila entre los 19 y 33 años, con una media de 25 años en ambos géneros; predominando, con un $64.9 \%$, los menores de 26 años en las mujeres, y los mayores de 27 años en los hombres con el $62.5 \%$.

El $32.4 \%$ de las mujeres presenta aseguramiento en salud en el régimen subsidiado, en los hombres el $12.5 \%$. La pertenencia étnica mayoritaria en las mujeres es la negra con un $55.4 \%$, mientras que en los hombres predomina la pertenencia étnica blanca con un $50.0 \%$ (Tabla 1).

El promedio de uso del CF en ambos géneros fue de 5, y el máximo de 15. Al comparar la frecuencia de uso del CF en las mujeres a partir del semestre IV al VII y del VIII al X con respecto a los hombres para volver a utilizar el CF presentan diferencias con una $\mathrm{p}<0.002$.

Todos los hombres, así como el $70 \%$ de las mujeres, consideran que se puede colocar antes de la relación; 
Tabla 1. Distribución de la información personal, Santiago de Cali 2015.

\begin{tabular}{lcccccc}
\hline & & \multicolumn{2}{c}{ Mujeres } & \multicolumn{2}{c}{ Hombres } & P \\
\hline & & Fr (\%) & IC (95\%) & Fr (\%) & IC (95\%) & \\
\hline Aseguramiento en Salud & Contributivo & $50(67.6 \%)$ & $55.7 \%-78-0 \%$ & $7(87.5 \%)$ & $47.3 \%-99.7 \%$ & 0.02 \\
\hline & Subsidiado & $24(32.4 \%)$ & $22.0 \%-44.3 \%$ & $1(12.5 \%)$ & $0.3 \%-52.7 \%$ & \\
\hline Pertenencia étnica & Indígena & $1(1.4 \%)$ & $0.0 \%-7.3 \%$ & $0(0.0 \%)$ & $0.0 \%-36.9 \%$ & \\
\hline & Mestiza & $3(4.1 \%)$ & $0.8 \%-11.4 \%$ & $1(12.5 \%)$ & $0.3 \%-52.7 \%$ & \\
\hline & Negra & $41(55.4 \%)$ & $43.4 \%-67.0 \%$ & $3(37.5 \%)$ & $8.5 \%-75.5 \%$ & \\
\hline Grupo de edad & Blanca & $29(39.2 \%)$ & $28.0 \%-51.2 \%$ & $4(50.0 \%)$ & $15.7 \%-84.3 \%$ & \\
\hline CF utilizados & $19-26$ & $48(64.9 \%)$ & $52.9 \%-75.6 \%$ & $3(37.5 \%)$ & $8.5 \%-75.5 \%$ & 0.1 \\
\hline & $27-33$ & $26(65.1 \%)$ & $24.4 \%-47.1 \%$ & $5(62.5 \%)$ & $24.5 \%-91.5 \%$ & \\
\hline & $<10$ & $37(50.0 \%)$ & $38.1 \%-61.9 \%$ & $3(37.5 \%)$ & $8.5 \%-75.5 \%$ & \\
\hline
\end{tabular}

Fuente: elaboración propia.

el $57.1 \%$ de las mujeres y el $75 \%$ de los hombres consideran positivo el material del cual está hecho el $\mathrm{CF}$, al funcionar para personas alérgicas al látex. Debido a que el CF se puede retirar hasta el final de la relación sexual, el 39.2\% de las mujeres y el $37.5 \%$ de los hombres aceptan como ventaja usar el CF (Tabla 2).

Tabla 2. Distribución de las ventajas en el uso del Condón Femenino

\begin{tabular}{|c|c|c|c|c|c|}
\hline \multirow[t]{2}{*}{ Ventajas } & \multicolumn{2}{|c|}{ Mujeres } & \multicolumn{2}{|c|}{ Hombres } & \multirow[t]{2}{*}{$\mathbf{P}$} \\
\hline & $\begin{array}{c}\text { SI } \\
\operatorname{Fr}(\%)\end{array}$ & $\begin{array}{l}\mathrm{NO} \\
\operatorname{Fr}(\%)\end{array}$ & $\begin{array}{l}\text { SI } \\
\operatorname{Fr}(\%)\end{array}$ & $\begin{array}{l}\text { NO } \\
\operatorname{Fr}(\%)\end{array}$ & \\
\hline 1.Se puede colocar antes de la relación. & $52(70.3 \%)$ & $22(29.7 \%)$ & $8(100.0 \%)$ & $0(0.0 \%)$ & - \\
\hline 2.No afecta la penetración. & $27(36.5 \%)$ & $47(63.5 \%)$ & $2(25.0 \%)$ & $6(75.0 \%)$ & 0.5 \\
\hline $\begin{array}{l}\text { 3.El material del cual está hecho funciona } \\
\text { para personas alérgicas al látex. }\end{array}$ & $38(57.1 \%)$ & $36(48.6 \%)$ & $6(75.0 \%)$ & $2(25.0 \%)$ & 0.2 \\
\hline 4.Es fácil de colocar. & $17(23.0 \%)$ & $57(77.0 \%)$ & $1(12.5 \%)$ & $7(87.5 \%)$ & 0.4 \\
\hline $\begin{array}{l}\text { 5.Se puede retirar hasta el final de la relación } \\
\text { sexual. }\end{array}$ & $29(39.2 \%)$ & $45(60.8 \%)$ & $3(37.5 \%)$ & $5(62.5 \%)$ & 0.9 \\
\hline 6.A mi pareja le gusta utilizarlo. & $11(14.9 \%)$ & $63(85.1 \%)$ & $0(0.0 \%)$ & $8(100.0 \%)$ & - \\
\hline $\begin{array}{l}\text { 7.Se puede colocar al inicio de la relación } \\
\text { sexual. }\end{array}$ & $27(36.5 \%)$ & $47(63.5 \%)$ & $1(12.5 \%)$ & $7(87.5 \%)$ & 0.01 \\
\hline $\begin{array}{c}\text { 8.Puede ser incorporado en los juegos } \\
\text { sexuales. }\end{array}$ & $13(17.6 \%)$ & $61(82.4 \%)$ & $3(37.5 \%)$ & $5(62.5 \%)$ & 0.1 \\
\hline 9.Su apariencia es agradable. & $6(8.1 \%)$ & $68(91.9 \%)$ & $0(0.0 \%)$ & $8(100.0 \%)$ & - \\
\hline 10.Aumentó el placer en la relación sexual. & $1(1.4 \%)$ & $73(98.6 \%)$ & $0(0.0 \%)$ & $8(100.0 \%)$ & - \\
\hline
\end{tabular}

Fuente: elaboración propia. 
En el grupo de las mujeres se consideran como mayores desventajas que el $54.1 \%$ de las parejas no les gusta utilizarlo, el 40.5\% manifestó dificultad para su colocación, y el $33.8 \%$ consideró que disminuye el placer sexual.

En el grupo de hombres, la disminución del placer en la relación sexual fue la desventaja más frecuente, así mismo, el $87.5 \%$ manifestó que a su pareja no le gusta utilizarlo, y un $62.5 \%$ informó que es difícil de colocar.

Al verificar con ambos géneros se presentan diferencias, al considerar que solo puede retirarse hasta el final de la relación sexual con $\mathrm{p}<0.005$, y se puede colocar antes de la relación sexual $\mathrm{p}<0.0007$ (Tabla 3 ).

Tabla 3. Distribución de las desventajas en el uso del Condón Femenino.

\begin{tabular}{|c|c|c|c|c|c|}
\hline \multirow[t]{2}{*}{ Desventajas } & \multicolumn{2}{|c|}{ Mujeres } & \multicolumn{2}{|c|}{ Hombres } & \multirow[t]{2}{*}{$\mathbf{P}$} \\
\hline & $\begin{array}{c}\text { SI } \\
\text { Fr }(\%)\end{array}$ & $\begin{array}{l}\mathrm{NO} \\
\operatorname{Fr}(\%)\end{array}$ & $\begin{array}{c}\text { SI } \\
\text { Fr }(\%)\end{array}$ & $\begin{array}{l}\text { NO } \\
\text { Fr }(\%)\end{array}$ & \\
\hline 1.Es difícil de colocar. & $30(40.5 \%)$ & $44(59.5 \%)$ & $5(62.5 \%)$ & $3(37.5 \%)$ & 0.2 \\
\hline 2.A mi pareja no le gusta utilizarlo. & $40(54.1 \%)$ & $34(45.9 \%)$ & $7(87.5 \%)$ & $1(12.5 \%)$ & 0.006 \\
\hline 3.Disminuyó el placer en la relación sexual. & $25(33.8 \%)$ & $49(66.2 \%)$ & $8(100.0 \%)$ & $0(0.0 \%)$ & - \\
\hline $\begin{array}{l}\text { 4.No puede ser incorporado en los juegos } \\
\text { sexuales. }\end{array}$ & $8(10.8 \%)$ & $66(89.2 \%)$ & $2(25.0 \%)$ & $6(75.0 \%)$ & 0.2 \\
\hline 5.Se puede retirar hasta el final de la relación & $2(2.7 \%)$ & $72(97.3 \%)$ & $2(25.0 \%)$ & $6(75.0 \%)$ & 0.005 \\
\hline $\begin{array}{l}\text { 6.Se puede colocar al inicio de la relación } \\
\text { sexual. }\end{array}$ & $3(4.1 \%)$ & $71(95.9 \%)$ & $2(25.0 \%)$ & $6(75.0 \%)$ & 0.018 \\
\hline $\begin{array}{l}\text { 7.Se puede colocar antes de la relación } \\
\text { sexual. }\end{array}$ & $1(1.4 \%)$ & $73(98.6 \%)$ & $2(25.0 \%)$ & $6(75.0 \%)$ & 0.000 \\
\hline 8.Interfiere con la penetración. & $9(12.2 \%)$ & $65(87.8 \%)$ & $1(12.5 \%)$ & $7(87.5 \%)$ & 0.9 \\
\hline
\end{tabular}

Fuente: elaboración propia.

Los participantes de ambos géneros consideran que durante la relación sexual con el CF no sintieron placer en relación con quienes sí lo afirman con una $\mathrm{p}<0.0009$. Tanto el $100 \%$ de los hombres como en el $86.5 \%$ de las mujeres les permitió sentir placer sin dificultad; la interrupción del acto sexual se dio en el $24.3 \%$ de las mujeres y en el $50 \%$ de los hombres. El $100 \%$ de los hombres y el $98.6 \%$ de las mujeres refieren no presentar ninguna diferencia entre usar el condón de látex masculino y el femenino (Tabla 4)

Tabla 4. Sensación durante la relación sexual con el uso del Condón Femenino.

\begin{tabular}{|c|c|c|c|c|c|}
\hline \multirow[t]{2}{*}{ Sensación durante la relación sexual } & \multicolumn{2}{|c|}{ Mujeres } & \multicolumn{2}{|c|}{ Hombres } & \multirow[t]{2}{*}{$\mathbf{P}$} \\
\hline & $\begin{array}{c}\text { SI } \\
\operatorname{Fr}(\%)\end{array}$ & $\begin{array}{c}\mathrm{NO} \\
\operatorname{Fr}(\%)\end{array}$ & $\begin{array}{c}\text { SI } \\
\operatorname{Fr}(\%)\end{array}$ & $\begin{array}{c}\mathrm{NO} \\
\operatorname{Fr}(\%)\end{array}$ & \\
\hline $\begin{array}{l}\text { 1.Le permitió sentir placer, pero fue difícil } \\
\text { acostumbrarse a la sensación de sentir el } \\
\text { condón. }\end{array}$ & $28(37.8 \%)$ & $46(62.2 \%)$ & $1(12.5 \%)$ & $7(87.5 \%)$ & 0.1 \\
\hline 2.Le permitió sentir placer sin dificultad & $10(13.5 \%)$ & $64(86.5 \%)$ & $0.0(\%)$ & $8(100.0 \%)$ & - \\
\hline $\begin{array}{l}\text { 3.Fue tan incómodo y desestimulante que se } \\
\text { interrumpió el acto sexual. }\end{array}$ & $18(24.3 \%)$ & $56(75.7 \%)$ & $4(50.0 \%)$ & $4(50.0 \%)$ & 0.1 \\
\hline 4.No le permitió sentir placer. & $2(2.7 \%)$ & $72(97.3 \%)$ & $3(37.5 \%)$ & $5(62.5 \%)$ & 0.00009 \\
\hline $\begin{array}{l}\text { 5.No hubo ninguna diferencia entre usarlo o } \\
\text { tener una relación sin protección. }\end{array}$ & $7(9.5 \%)$ & $67(90.5 \%)$ & $0.0(\%)$ & $8(100.0 \%)$ & - \\
\hline $\begin{array}{l}\text { 6.No hubo ninguna diferencia entre usar el } \\
\text { condón de látex masculino y el femenino. }\end{array}$ & $1(1.4 \%)$ & $72(98.6 \%)$ & $0.0(\%)$ & $8(100.0 \%)$ & - \\
\hline
\end{tabular}

Fuente: elaboración propia. 
de los hombres presentaron inconformidad con la

El 50\% de los hombres manifestó satisfacción después de haber utilizado el $\mathrm{CF}$, el $50 \%$ de ambos géneros 10 experiencia. (Tabla 5).

consideran seguro; el $24.3 \%$ de las mujeres y el $50 \%$

Tabla 5. Sensación después del uso del Condón Femenino.

\begin{tabular}{cccccc|}
\hline Sensación después & \multicolumn{2}{c}{ Mujeres } & \multicolumn{2}{c}{ Hombres } & P \\
\hline & SI & NO & SI & NO & 0.1 \\
\hline 1.Satisfecho por la experiencia. & $19(25.7 \%)$ & $55(74.3 \%)$ & $4(50.0 \%)$ & $4(50.0 \%)$ & 0.006 \\
\hline 2.Seguro por la protección del condón & $37(50.0 \%)$ & $37(50.0 \%)$ & $4(50.0 \%)$ & $4(50.0 \%)$ & 0.1 \\
\hline 3.Inconforme por la experiencia. & $18(24.3 \%)$ & $56(75.7 \%)$ & $4(50.0 \%)$ & $4(50.0 \%)$ & 0.4 \\
\hline 4.Insegura de la protección del condón. & $4(5.4 \%)$ & $70(94.6 \%)$ & $1(12.5 \%)$ & $7(87.5 \%)$ & - \\
\hline 5.Ninguna. & $2(2.7 \%)$ & $72(97.3 \%)$ & $0(0.0 \%)$ & $8(100.0 \%)$ & - \\
\hline $\begin{array}{c}\text { 6.Satisfecho en lo personal, pero la pareja } \\
\text { refiere insatisfacción. }\end{array}$ & $14(18.9 \%)$ & $60(81.1 \%)$ & $0(0.0 \%)$ & $8(100.0 \%)$ & - \\
\hline
\end{tabular}

Fuente: elaboración propia.

La aceptación para el uso del CF en todas las relaciones de pareja no presenta diferencias en ambos géneros; sin embargo, el $54.1 \%$ de las mujeres y el $25 \%$ de los hombres con una $\mathrm{p}<0.1$ dejan la posibilidad de continuar con su uso.

\section{Discusión}

Las ventajas descritas por los participantes del estudio en la utilización del CF en el $70 \%$ de las mujeres expresan que se puede colocar antes de la relación en una proporción mayor a la descrita en los estudios de España (1) y China (6). En el estudio el 57.1\% considera positivo el material del cual está hecho el $\mathrm{CF}$ al funcionar para personas alérgicas al látex, y con relación a que se puede retirar hasta el final de la relación sexual en un $39.2 \%$, encontrando diferencias con lo presentado por estudios africanos $(7,8)$, y lo referenciado en el Salvador y Nicaragua en un estudio con trabajadoras sexuales, donde las mujeres reportaron que el diseño de los $\mathrm{CF}$ dificultaba el aprendizaje de su inserción y remoción (24).

Otros aspectos relacionados en el estudio fue el miedo con respecto a la utilización del CF referido por el $51.4 \%$ de las mujeres, el dolor $8.1 \%$, ardor $13.5 \%$ y vergüenza $9.5 \%$, los cuales podrían intervenirse con mayor acercamiento a las mujeres y profundización en los contenidos de las estrategias educativas. Comparado con una población de Costa Rica donde estos aspectos se presentaron en menor proporción siendo el miedo en las mujeres del $12.5 \%$, dolor $1.3 \%$, ardor $3.8 \%$ y vergüenza $8.8 \%$, situación que debe ser profundizada en otros estudios (9).

Las mujeres consideran como mayores desventajas comparado con los hombres, que a la pareja no le gusta utilizarlo en un $54.1 \%$, este hallazgo puede estar relacionado con la poca disponibilidad y empleo del método, comparado con lo encontrado en Ecuador (25), Salvador - Nicaragua (24) y Brasil (26), donde las mujeres que fueron a más controles terminaron usándolos. En los aspectos señalados como desventajas con el uso del CF se plantea la disminución del placer en la relación sexual, a la pareja no le gusta utilizarlo en un $87.5 \%$, y el $62.5 \%$ expresa que es difícil de colocar. Estos resultados pueden tener el sesgo de que no se tuvo una comunicación directa ni capacitación con las parejas, y fueron ellos los que de manera independiente y unilateral decidieron vivir la experiencia comprometiendo a sus compañeras sexuales.

Trabajar con hombres antes que con las parejas de sexo femenino muestra resultados muy diferentes. En Zimbabwe, Nigeria y Camerún, 336 hombres participaron en grupos focales, en donde las ventajas percibidas de $\mathrm{CF}$ con respecto a otros métodos de protección se relacionaron con placer, eficacia y ausencia de efectos secundarios (6). Los hombres solteros y casados prefieren el uso de los condones femeninos con fines de anticoncepción en lugar de protección frente a las infecciones de transmisión sexual. 
Con respecto a las ventajas en el estudio realizado, todos los hombres describen que se puede colocar antes de la relación, el 75\% considera positivo el material del cual está hecho al funcionar para personas alérgicas al látex, siendo similares los resultados frente a las mujeres.

A pesar de tener los condones femeninos más de 20 años en el mercado internacional, la mayoría de los participantes y sus parejas no conocían este método de protección para las mujeres.

La aceptación para el uso del CF en todas las relaciones de pareja no presenta diferencias en ambos géneros; sin embargo, dejan la posibilidad de continuar con el $25.9 \%$ de los hombres participantes. Estos resultados están en consonancia con la encuesta Nacional de Demografía y Salud en donde si bien se han tenido avances importantes frente al uso del condón masculino, en la última relación sexual apenas alcanza un $30 \%$ en la población joven, en este sentido no basta con el conocimiento y disponibilidad para garantizar su uso (13). Es necesario generar nuevas y novedosas estrategias de intervención que lleven a los jóvenes a optar por este tipo de método, que les permita negociar en pareja sus formas de prevención de enfermedades y la planificación familiar (17).

Con los riesgos actuales de la salud asociados a la vida sexual y reproductiva dentro del concepto de gestión del riesgo, cobra gran importancia impulsar, sobre todo entre la población joven, este método de barrera para el autocuidado de las mujeres y sus parejas, a la vez que se avanza con el enfoque de género definido desde el Plan Decenal de Salud Pública (16). Una de las formas de garantizar su aceptación y empoderamiento por parte de las mujeres es, sin duda, reconocer con anterioridad las percepciones que tienen los hombres y las mujeres frente al CF, para fortalecer los aspectos positivos y reflexionar sobre los que se consideran negativos, teniendo en cuenta las particularidades de la población con un enfoque diferencial.

En el caso de Colombia, si bien es cierto que no se ha contado con el acceso y disponibilidad permanente en el mercado de los CF, han sido distribuidos prioritariamente desde los servicios amigables para jóvenes de manera intermitente (17), previa capacitación dada por los funcionarios, sin que hasta el momento se les realice ningún tipo de seguimiento que permita conocer qué piensan los adolescentes y jóvenes, y si están dispuestos a utilizarlos de manera continua durante su vida sexual.

Como lo afirman Guerrero et al. (2018), es necesario tener una utilización informada para el método de protección en la salud sexual y reproductiva como es el CF (27), y el disfrute de la sexualidad sin riesgos, con adquisición de conocimientos y entrenamiento conductual previo para evitar errores en el empleo, de acuerdo con las evidencias obtenidas en el estudio de adolescentes por Landa y colaboradores (28).

El estudio presenta limitaciones al ser desarrollado solo en un sector específico de estudiantes, además, en el caso del grupo de los hombres fueron ellos quienes recibieron la información de primera mano y sus respectivas compañeras solo contaron con información secundaria proporcionada por los participantes.

\section{Conclusiones}

La utilización del $\mathrm{CF}$ es una alternativa viable para tener sexo seguro, los hombres presentan mayor rechazo al verse afectado el placer sexual. Sin embargo, la aceptación del CF está mediada por el disfrute de la sexualidad, el ejercicio de los derechos sexuales y reproductivos, la elección de estrategias de prevención de ITS y embarazos no deseados, las experiencias y expectativas personales.

El CF como método de barrera es una posibilidad para que las mujeres libremente informadas puedan optar por él. Proporcionar información sobre los condones y garantizar la accesibilidad económica-administrativa no es suficiente, sino se toman en cuenta todos los factores que facilitan o dificultan su aceptación. El CF puede contribuir a complementar los métodos de planificación familiar ya existentes, pero además ofrece la posibilidad de complementar lo avanzado con el condón masculino como método de barrera, sobre todo para reducir los casos de ITS.

\section{Agradecimientos}

Se agradece a las personas participantes en el estudio.

\section{Conflictos de intereses}

Los autores declaran no tener conflictos de intereses.

\section{Financiación}

Propia

\section{Referencias}

1. Lameiras M, Ricoy MC, Carrera MV, Failde JM, Núñez AM. Evaluación del uso del preservativo femenino promovido desde un programa de educación para la salud: un enfoque cualitativo. Saude soc. [Internet]. Junio de 2011 [Consultado en abril de 
2018]; 20 (2): 410-424. Disponible en: http://dx.doi. org/10.1590/S0104-12902011000200013

2. Organización Mundial de la Salud. Criterios médicos de elegibilidad para el uso de anticonceptivos. Un pilar de la OMS para la planificación familiar. 4 ed. OMS; 2012. 89-96. Disponible en: http://apps.who.int/iris/ bitstream/handle/10665/44710/9789243563886 spa. pdf? sequence $=1$

3. Organización Mundial de la Salud UNFPA, UNAIDS. Preservativos femeninos: Especificaciones generales, precalificación y directrices de adquisición, 2012. [Internet] 2012 [Consultado enero 2017]. Disponible en: https://www.unfpa.org/sites/default/files/resource-pdf/ Preservativos Femininos Escpecificaciones Generales. pdf

4. ONUSIDA. Informe mundial: informe sobre la epidemia mundial de Sida [Internet] 2013 [Consultado mayo 2017]. Disponible en: https://www.unaids.org/ sites/default/files/media_asset/UNAIDS_Global_ Report 2013 es 1.pdf

5. ONUSIDA. Estrategia ONUSIDA 2016-2021. Acción acelerada para acabar con el sida [Internet] 2019 [Consultado diciembre 2019]. Disponible en: https://www.unaids.org/sites/default/files/media asset/UNAIDS-strategy-2016-2021 es.pdf

6. Wang X, Liu M, Cheng Y. Acceptability of Phoenurse female condom and second-generation Femidom female condom in Chinese women. J Obstet Gynaecol Res. [Internet] 2016 [Consultado en April 2018];42(11):15671574. https://doi.org/10.1111/jog. 13080

7. Koster W, Bruinderink MG, Janssens W. Empowering Women or Pleasing Men.Analyzing Male Views on Female Condom Use in Zimbabwe, Nigeria and Cameroon. Int Perspect Sex Reprod Health. [Internet] 2015 Sep [Consultado en Junio 2018]; 41 (3): 126-35. https://doi.org/10.1363/4112615

8. Mogale R, Mulaudzi FM, Peu MD, et al. The constraints and concerns regarding the size and/or shape of the second-generation female condom: The narratives from the healthcare providers. Afr J Prm Health Care Fam Med. [Internet] 2016 [Consultado en Abril 2017]; 8(2), 1-7. https://doi.org/10.4102/phcfm. v8i2.1146

9. Fondo de Población de las Naciones Unidas (UNFPA)Seguro Social Costa Rica. Estudio de aceptabilidad del condón femenino en población transfronteriza habitante de Upala y población urbana de San José. [Internet] Mayo, 2012. [Consultado en junio 2017]. Disponible en: https://costarica.unfpa.org/sites/ default/files/pub-pdf/estudio-aceptabilidad-condonfemenino-migrantes.pdf

10. Chisaguano-Adame JP. Conocimiento y uso del preservativo femenino como estrategia de prevención de las infecciones de transmisión sexual en las estudiantes de la carrera de enfermería de la universidad técnica de Ambato, período junio - diciembre 2014. Universidad Técnica de Ambato - Facultad de Ciencias de la Salud - Carrera de Medicina. Abril, 2015. Disponible en: http://repositorio.uta.edu.ec/ jspui/handle/123456789/9424

11. Bolaños M. Barreras para el acceso y el uso del condón desde la perspectiva de género. Horiz. sanitario [revista en la Internet]. 2019 Abr [citado 2020 septiembre 05]; 18( 1 ): 67-74. https://doi.org/10.19136/hs.a18n1.2306

12. Minsalud, Profamilia. Encuesta Nacional de Demografía y Salud 2010. Profamilia. Colombia, 2011. 120-124. Disponible en https://dhsprogram. com/pubs/pdf/fr246/fr246.pdf

13. Ministerio de Salud y Protección Social, Profamilia. Encuesta Nacional de Demografía y Salud. Resumen Ejecutivo. Colombia, 2015. 43-58.

14. Sabogal AL, Apolinar J, Peralta Carvajal C, Martínez Montañez LM. Infecciones de Transmisión Sexual Grupo Transmisibles Instituto Nacional de Salud. Protocolo de Vigilancia en Salud Pública Sífilis Gestacional y Sífilis Congénita. [Internet] Colombia, Bogotá, 2015 [Consultado en mayo de 2018]. Disponible en: https://www.minsalud.gov.co/sites/ rid/Lists/BibliotecaDigital/RIDE/IA/INS/protocolovigilancia-sifilis-gestacional.pdf

15. Ministerio de Salud y Protección Social. Resolución 0518 de 2015 [Internet]. Colombia, Bogotá, 2015. [Consultado en mayo de 2016]. Disponible en: $\quad$ https://www.minsalud.gov.co/sites/rid/Lists/ BibliotecaDigital/RIDE/DE/DIJ/resolucion-0518de-2015.pdf

16. Ministerio de Salud y Protección Social. Plan Decenal de Salud Pública 2012-2021 [Internet]. Colombia, Bogotá, 2013. [Consultado en mayo de 2018]. Disponible en: https://www.minsalud.gov.co/sites/rid/ Lists/BibliotecaDigital/RIDE/VS/ED/PSP/PDSP.pdf

17. Ministerio de Salud y Protección Social, Fondo de Población de las Naciones Unidas - UNFPA. Análisis de situación de condones en Colombia 2015 [Internet]. Colombia, Bogotá, 2015. [Consultado en mayo de 2016]. Disponible en: https://colombia.unfpa.org/sites/ default/files/pub-pdf/analisiscondonesColombia web.pdf

18. World Health Organization, UNFPA. Scientific and technical requirements to formulate a female condom generic specification and prequalification scheme. Female condom technical review committee report. [Internet] 2012 [Consultado en Abril 2018] Available in: http://apps.who.int/iris/bitstream/10665/70838/1/ WHO RHR 1203 eng.pdf

19. Ministerio de Salud y Protección Social, Fondo de Población de las Naciones Unidas. Guía del uso del condón femenino. [Internet] Bogotá D.C, 
2014. Disponible en: http://colombia.unfpa.org/es/ publicaciones/guia-de-uso-del-cond $\% \mathrm{C} 3 \% \mathrm{~B} 3 \mathrm{n}$ femenino?page $=3 \% 2 \mathrm{C} 0 \% 2 \mathrm{C} 7$

20. Beksinska, M, Smit J, Joanis C, Usher-Patel M, Potter W. Female condom technology: new products and regulatory issues. Contraception, [Internet] 2011 [Consultado en Mayo 2018]; 83(4):316-321. https:// doi.org/10.1016/j.contraception.2010.07.022

21. Fundación Desafío. Estudio cualitativo en mujeres jóvenes urbanas, amas de casa y sus parejas de la ciudad de Quito, sobre percepciones e intención de uso del condón femenino [Internet] Quito Ecuador, 2008 [Consultado en mayo de 2018]. Disponible en: http://www.sidastudi.org/es/registro/d25f08042719e3 $\underline{5601278525627500 \mathrm{bb}}$

22. Asociación Médica Mundial. Declaración de Helsinki de la Asociación Médica Mundial. 41 ${ }^{\mathrm{a}}$ Asamblea Médica Mundial; 1989. Disponible en: https:// www.wma.net/wp-content/uploads/2018/07/DoHSept1989.pdf

23. República de Colombia. Ministerio de Salud. Resolución No. 008430 de 1993. Por la cual se establecen las normas científicas, técnicas y administrativas para la investigación en salud. Bogotá DC. Min salud. 1993. Disponible en: https:// www.minsalud.gov.co/Normatividad Nuevo/ RESOLUCION\%208430\%20DE\%201993.pdf

24. Mack N, Grey TG, Amsterdam A, Williamson N, Interiano - Matta Cl. Introducción del uso de los condones femeninos entre las trabajadoras sexuales en América Central. Perspectivas Internacionales en Salud Sexual y Reproductiva. [Internet] 2011 [Consultado en junio 2018], pp. 31-39. Disponible en: http://www.sidastudi.org/es/registro/ ff808181539e2b3901543c9d5dbc040f

25. Yánez-Ruiz M. Barreras para la utilización del condón femenino en la población de mujeres en edad fértil que acuden al centro de salud Cevallos. [Tesis de Medicina] Universidad Técnica de Ambato. Ambato-Ecuador 19-ago-2014. Disponible en: http:// repositorio.uta.edu.ec/handle/123456789/7983

26. Oliveira FS, Silva da Costa CF, Pereira da Costa Kerber, N, Mendes de Barros A, Wachholz V. A., et al. El uso del preservativo femenino por las profesionales del sexo. Enfermería Global, [Internet] 2012. [Consultado junio de 2018]; 11(26), 390-398. https://doi.org/10.4321/S1695-61412012000200024

27. Guerrero N, PérezM,Alfonso CA, PérezR,Alfonso M, Bautista L. Estudio sobre la aceptación de los condones femeninos: fase exploratoria. Revista Sexología y Sociedad. [Internet] 2018 [Consultado enero de 2019]; 24(2):102-111. http://revsexologiaysociedad.sld.cu/ index.php/sexologiaysociedad/article/view/654

28. Landa E, Quiñones SI, Flores AD, Martínez AE, Sánchez JJ. Entrenamiento conductual del uso correcto del condón femenino en adolescentes: un estudio aleatorizado. Psicología y Salud. [Internet] 2018[Consultado enero de 2019]; 28(1):25-35. https:// doi.org/10.25009/pys.v28i1.2543 